\title{
Polio Vaccination
}

National Cancer Institute

\section{Source}

National Cancer Institute. Polio Vaccination. NCI Thesaurus. Code C96409.

The administration a series of either live or inactivated poliovirus for the prophylaxis of poliomyelitis. 